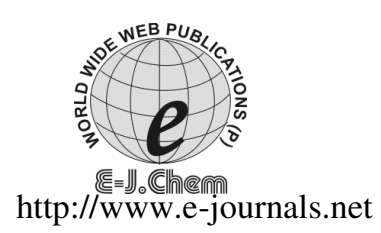

ISSN: 0973-4945; CODEN ECJHAO
E-Journal of Chemistry
$2011, \mathbf{8 ( 4 )}, 1522-1527$

\title{
Synthesis and Microbiological Activity of Some Newly Synthesized Derivatives of 2-Oxo-2H-chromen-2-one
}

\author{
MAJLINDA DACI-AJVAZI ${ }^{\S}$, SEVDIJE GOVORI ${ }^{\S *}$ and SHUHRETA OMERAGIQ \\ ${ }^{\S}$ University of Prishtina \\ Faculty of Natural and Mathematical Sciences \\ Department of Chemistry, 10000 Prishtina, Republic of Kosovo \\ Microbiological Laboratory of Regional Water Company \\ s_govori@yahoo.com
}

Received 28 September 2010; Accepted 27 November 2010

\begin{abstract}
By the action of 2-amino-5-methylthio-1,3,4-thiadiazole, 3-amino-5methylisoxazole, 2-amino-6-fluorobenzothiazole, 2-amino-5-chloropyridine, respectively, on 4-chloro-2-oxo- $2 \mathrm{H}$-chromene-3-sulfonyl chloride, the corresponding 9-methylthio-7,7-dioxo-7,7a-dihydro-5-oxo-7 $\lambda^{6}, 10$-dithia-8,11diaza-cyclopenta[b] phenantren-6-one, 9-methyl-7,7-dioxo-7H-5,8-dioxa-7 $\lambda^{6}$ thia-7a,11-diaza-cyclopenta[b] phenantren-6-one, 9-fluoro-7,7-dioxo-7 $\mathrm{H}$-5oxa-7 $7 \lambda^{6}, 12$-dithia-7a,13-diaza-indeno[1,2-b] phenantren-6-one and 9-Chloro7,7-dioxo-7H-5-oxa- $7 \lambda^{6}$-thia-7a,12-diaza-benzo[ $\left.\alpha\right]$ anthracen -6-one were formed and they have been isolated in satisfying yields. Based on the biological activity of chromene-2-ones and heterocyclic compounds condensed in position 3 and 4, we also studied microbiological activity of these new compounds (5-8), against Staphylococcus aureus ATCC 25923, Streptococcus pneumoniae, Aeromonas Salmonicida, Bacillus spp and some of them exhibited significant activity.
\end{abstract}

Keywords: 2-Oxo-2H-chromen-2-one, Heterocyclic amines, Synthesis, Microbiological activity

\section{Introduction}

4-Hydroxy- $2 H$-chromen-2-one has evoked a great deal of interest due to their biological properties and characteristic conjugated molecular architecture. In continuation of our work ${ }^{1,2}$ on the chemistry of 3,4-disubstituted-2-oxo- $2 \mathrm{H}$-chromene and the derived coumarins annelated in the 3,4-position, we decided to prepare some new 2-oxo- $2 \mathrm{H}$-chromene derivatives with a variety of nucleophiles, where we produced a number of novel cyclised coumarins. Hard and borderline nucleophiles exclusively substituted chlorine in position 4, while soft nucleophiles substituted the group in position 3. Chromene-2-one derivatives have been reported for anticoagulant ${ }^{3-5}$, antibacterial ${ }^{6-7}$, antiinflamatory ${ }^{8}$, antimicrobial ${ }^{9}$, antiHIV ${ }^{10-13}$, 
antioxidant ${ }^{14}$, anticancer ${ }^{15}$ and antiproliferative and antiviral ${ }^{16}$ activities. It was found that when one biodynamic heterocyclic system was coupled with another heterocyclic system, enhanced biological activity was produced.

These newly synthesized polycyclic compounds (5-8), have similar structure with lots of chromene-2-one compounds that are well known for their physiological activity ${ }^{17-19}$. From all chromene-2-ones, 4-hydroxychromene-2-one differs for its chemical reactivity, as it contains the enolysed $\beta$-ketoesteryc system. The molecule of 4-hydroxychromene-2-one contains two cycles: the benzene and the $\alpha$-pyrone one-which has the $-\mathrm{OH}$ group attached in the -4 position. This $\alpha$-pyrone nucleus, reacts faster than benzene one, with either electrophylic or nucleophylic reagents.

Therefore, 4-hydroxychromene-2-one, enable possibilities for synthesis of new condensed and cyclisized derivatives, enabling transition from bicyclic to heteropolycyclic compounds.

\section{Experimental}

Melting points of synthesized compounds (5-8) were determined with a Buechi apparatus and are uncorrected. IR spectra $\left(\mathrm{KBr}\right.$ in $\left.\mathrm{cm}^{-1}\right)$ were recorded on Perkin Elmer Spectrum BX FTIR spectrophotometer, ${ }^{1} \mathrm{H}$ NMR were recorded on Bruker Avance DPX 300 spectrophotometer at $300 \mathrm{MHz}$ for ${ }^{1} \mathrm{H}$, using DMSO-d6 and $\mathrm{CDCl}_{3}$ as solvent, with TMS as the internal standard (chemical shifts in $\delta \mathrm{ppm}$ ). The purity of the compounds was monitored by TLC using precoated HF 254 (60) silica gel plates.

\section{General methods of synthesis}

4-Hydroxychromene-2-one $(1,16 \mathrm{~g})$ was added in one portion to monochlorosulfonic acid $(40 \mathrm{~mL})$. Mixture was refluxed in water bath in $70{ }^{\circ} \mathrm{C}$ for 2.5 hours, then $125 \mathrm{~mL}$ dry benzene was added drop wise to the mixture and after short vigorous mixing (20 min), solution was left for short period in a room temperature and very voluminous precipitate was formed. Precipitate (2) was filtered on a glass funnel and washed first with dioxane $(20 \mathrm{~mL})$ then with ethylacetate $(20 \mathrm{~mL})$ and then with ether $(20 \mathrm{~mL})$. After washing we obtained a white powder (93\% yield). Ammonium salt of 4-hydroxychromene-2-one-3-sulfonic acid $(2,14 \mathrm{~g})$ was dissolved in small quantity of water and with vigorous mixing $\mathrm{KOH}(4 \%, 100 \mathrm{~mL})$ was added. Rapidly formed white crystals (3, yield $86 \%$ ) were filtered and washed off with ethanol (95\%). $8 \mathrm{~g}$ of 3 was added to $\mathrm{POCl}_{3}(60 \mathrm{~mL})$ and refluxed in a hot oily bath for $4 \mathrm{~h}$. Hot mixture was filtered off in a vacuum filter, filtrate was evaporated and obtained yellow crystal precipitate $(4$, yield $30 \%)$.

$1 \mathrm{~g},(0.0038 \mathrm{~mol})$ of 4-chloro-2-oxo-2H-chromene-3-sulfonyl chloride (4) was dissolved in $20 \mathrm{~mL}$ of dry benzene and an appropriate equivalent portion of heterocyclic amino derivatives was added, in presence of triethylamine $(2 \mathrm{~mL})$, as catalyst and the mixture was refluxed for 4-10 h. After cooling, the precipitate was filtered off, washed with hot benzene and dried.

9-Methylthio-7,7-dioxo-7,7a-dihydro-5-oxo-7 $\lambda^{6}, 10$-dithia-8,11-diaza-cyclopenta[b] phenantren-6-one (5)

A mixture of 4-chloro-2-oxo-2H-chromene-3-sulfonyl chloride $(1 \mathrm{~g}, 0.0036 \mathrm{~mol}), 20 \mathrm{~mL}$ of dry benzene, $0.53 \mathrm{~g},(0.0036 \mathrm{~mol})$ of 2-amino-5-methylthio-1,3,4-thiadiazole and a portion of thriethylamine were refluxed for $4 \mathrm{~h}$. The solution was filtered, washed off with benzene then crystallized from ethanol. The obtained product was white color. 
Yield 35,62\% M.P. $135{ }^{\circ} \mathrm{C}$, IR (KBr) $\left(\mathrm{v} \mathrm{cm}^{-1}\right): 3800-3153(\mathrm{OH}, \mathrm{NH}) ; 2920(\mathrm{C}-\mathrm{H} \mathrm{al})$; 1703(C=O, $\alpha$-pir); 1621(C=N); 1562-1356(C=Car); 1207-1161 $\left(\mathrm{SO}_{2}\right) ; 751(\mathrm{C}-\mathrm{Car}) ; 648(\mathrm{C}-\mathrm{S}-\mathrm{C})$; ${ }^{1} \mathrm{H}-\mathrm{NMR}$ (DMSO-d6) (ppm): $14.1 \mathrm{~s}(1 \mathrm{H}, \mathrm{OH}) ; 7.88-5.75 \mathrm{~m}$ (4Har); $4.2 \mathrm{~s}(1 \mathrm{H}, \mathrm{NH}) ; 3.14-2.49 \mathrm{~s}$ $\left(3 \mathrm{H}, \mathrm{CH}_{3}\right) \mathrm{C}_{13} \mathrm{H}_{11} \mathrm{O}_{5} \mathrm{~N}_{3} \mathrm{~S}_{3}$ : C 40.51, H 2.87, N 10.90, Exp: C 39.50, H 2.90, N $10.40 \mathrm{M}^{+}$teo: 385,43; $\mathrm{M}^{+} \exp : 385.43 . \mathrm{M}_{\left(\mathrm{C}_{13} \mathrm{H}_{11} \mathrm{O}_{\left.5 \mathrm{~N}_{3} \mathrm{~S}_{3}\right)}\right)} 385$ (7.2), 368 (21.5), 242 (88.7), 162 (93), 120 (100), 74 (46).

9-Metil-7,7-dioxo-7H-5,8-dioxo-7 $\lambda^{6}$-tia-7a,11-diaza-ciklopenta[b]phenantren-6-one (6) A mixture of 4-chloro-2-oxo- $2 \mathrm{H}$-chromene-3-sulfonyl chloride $(1 \mathrm{~g}, 0.0036 \mathrm{~mol}), 20 \mathrm{~mL}$ of dry benzene, $0.34 \mathrm{~g}(0.0036 \mathrm{~mol})$ of 3-amino-5-methyl-izoxazole and a portion of thriethylamine were refluxed for $10 \mathrm{~h}$. The solution was filtered, washed off with benzene than crystallized from ethanol. The obtained product was yellowish color.

Yield 29.1\% M.P. $242.3{ }^{\circ} \mathrm{C}$, IR (KBr) $\left(\mathrm{v} \mathrm{cm}^{-1}\right)$ : 3030(C-Har); 2950(C-Hal); 1715(C=O, $\alpha$-pir); 1609(C=N); 1551-1433(C=Car); 1270-1178(SO2); 762(C-Car); ${ }^{1} \mathrm{H}-\mathrm{NMR}$ (DMSO-d6) (ppm): 8.33-7.15 m (4H, ar); $1.7 \mathrm{~s}\left(3 \mathrm{H}, \mathrm{CH}_{3}\right) . \mathrm{C}_{13} \mathrm{H}_{8} \mathrm{O}_{5} \mathrm{~N}_{2} \mathrm{~S}: \mathrm{C} 51.32, \mathrm{H} 2.65, \mathrm{~N}$ 9.21, Exp: C 51.14, H 2.88, N 9.05 M+teo: 304,28 M+exp: 305.0, $\mathrm{M}_{\left(\mathrm{C} 13 \mathrm{H} 8 \mathrm{O}^{+} \mathrm{N} 2 \mathrm{~S}\right)}: 305$ (67), 289 (8.2), 154 (100), 137 (72), 71 (90.2), 55 (80).

\section{9-Fluoro-7,7-dioxo-7H-5-oxa-7 $\lambda^{6}, 12$-dithia-7a,13-diaza-indeno[1,2-b]phenantren- 6-one (7)}

A mixture of 4-chloro-2-oxo- $2 \mathrm{H}$-chromene-3-sulfonyl chloride $(1 \mathrm{~g}, 0.0036 \mathrm{~mol}), 20 \mathrm{~mL}$ of dry benzene, $0.62 \mathrm{~g}(0.0036 \mathrm{~mol})$ of 2-amino-6-fluorobenzothiazole and a portion of thriethylamine were refluxed for $10 \mathrm{~h}$. The solution was filtered, washed off with benzene than crystallized from ethanol. The obtained product was white color.

Yield (31.34\%), M.P. $254-256{ }^{\circ} \mathrm{C}$, IR $(\mathrm{KBr})\left(\mathrm{v} \mathrm{cm}^{-1}\right)$ : 3070(C-Har); $1710(\mathrm{C}=\mathrm{O}, \alpha$-pir $)$; 1608(C=N); 1570-1469(C=Car); 1269-1170( $\left.\mathrm{SO}_{2}\right) ; 759(\mathrm{C}-\mathrm{Car}) ;{ }^{1} \mathrm{H}-\mathrm{NMR}$ (DMSO-d6) (ppm): 8.36-7.30 m (7H, arom). $\mathrm{C}_{16} \mathrm{H}_{7} \mathrm{O}_{4} \mathrm{~N}_{2} \mathrm{~S}_{2} \mathrm{~F}$ : C 51.33, H 1.88, N 7.48, Exp: C 51.00, H 2.26, N $7.43 \mathrm{M}^{+}$teo: 374.36, $\mathrm{M}^{+}$exp: 375.0, $\mathrm{M}_{(\mathrm{C} 16 \mathrm{H} 7 \mathrm{O} 4 \mathrm{~N} 2 \mathrm{~S} 2 \mathrm{~F})} 375$ (23), 307 (25.4), 289 (12), 154 (100), 137 (72), 71 (40), 55 (47).

\section{9-Chloro-7,7-dioxo-7H-5-oxa-7 $\lambda^{6}$-thia-7a,12-diaza-benzo[ $\left.\alpha\right]$ anthracen-6-one (8)}

A mixture of 4-chloro-2-oxo- $2 H$-chromene-3-sulfonyl chloride $(1 \mathrm{~g}, 0.0036 \mathrm{~mol}), 20 \mathrm{~mL}$ of dry benzene, $0.46 \mathrm{~g}(0.0036 \mathrm{~mol})$ of 2-amino-5-chloropyridine and a portion of thriethylamine were refluxed for $10 \mathrm{~h}$. The solution was filtered, washed off with benzene than crystallized from ethanol. The obtained product was intensive yellow color.

Yield $(28,0 \%)$, M.P. $>270{ }^{\circ} \mathrm{C}$, IR $(\mathrm{KBr})\left(\mathrm{v} \mathrm{cm}^{-1}\right): 1715(\mathrm{C}=\mathrm{O}, \alpha-\mathrm{pir}) ; 1627(\mathrm{C}=\mathrm{N}) ; 1547-$ 1447(C=Car); 1263-1122(SO2); 755(C-Car); ${ }^{1} \mathrm{H}-\mathrm{RBM}$ (DMSO-d6) (ppm): 8.17-7.06 m (7H, arom). ${ }^{13} \mathrm{C}-\mathrm{RBM}$ (DMSO-d6) (40.33-41.24) $153.67(\mathrm{C}=\mathrm{N}) ; 138.11$ (Car); 116.72 (C-Cl);115.4 (C-SO $)_{2}$. $\mathrm{C}_{14} \mathrm{H}_{7} \mathrm{O}_{4} \mathrm{~N}_{2} \mathrm{SCl}: \mathrm{C}$ 50.23, H 2.108, N 8.37, Exp: C 49.95, H 2.24, N 8.15. $\mathrm{M}^{+}$teo: 334,72; $\mathrm{M}^{+}$exp: 335.0; $\mathrm{M}_{(\mathrm{C} 14 \mathrm{H} 7 \mathrm{O} 4 \mathrm{~N} 2 \mathrm{SCl})} 335$ (82.9), 307 (23), 289 (11), 154 (100), 137 (75.8), 71 (71), 55 (61).

\section{Results and Discussion}

4-Chloro-2-oxo-2H-chromene-3-sulfonyl chloride (4) was prepared by the series of reactions of 4-hydroxy-chromene-2-one with $\mathrm{ClSO}_{3} \mathrm{H}$, with $\mathrm{NH}_{3}$, then with $4 \% \mathrm{KOH}$ solution, and with $\mathrm{POCl}_{3}$. New compounds (5-8) were obtained in reaction of (4) with amino derivatives of heterocyclic compounds in presence of triethylamine. 


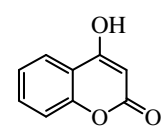

(1)

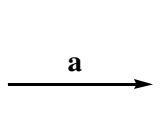

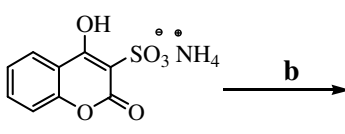

(2)<smiles>O=c1oc2ccccc2c(O)c1[14CH2][Na]</smiles>

(3)<smiles>O=C(Cl)c1c(Cl)c2ccccc2oc1=O</smiles>

(4)

Figure 1. Synthesis of 4-chloro-2-oxo-2H-chromene-3-sulfonyl chloride (4) Reagents: (a) $\mathrm{ClSO}_{3} \mathrm{H}, \mathrm{NH}_{3}$ (b) $4 \% \mathrm{KOH}$, (c) $\mathrm{POCl}_{3}, \mathrm{DMF}$<smiles>O=c1oc2ccccc2c(Cl)c1S(=O)(=O)Cl</smiles>

(4)<smiles></smiles>

(5-8)<smiles>O=c1oc2ccccc2c(Cl)c1Nc1cn[Y]n1</smiles><smiles>O=c1oc2ccccc2c(NCc2cncnc2)c1O</smiles><smiles></smiles>

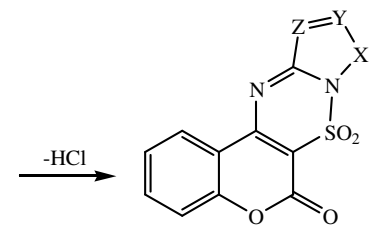

(5-8)

Figure 2. Reaction mechanism<smiles>CC1=NN2Oc3c(c4ccccc4oc3=O)N=C2S1</smiles>

(5)<smiles>Cc1cc2nc3c4ccccc4oc(=O)c3n2o1</smiles>

(6) 
<smiles>O=c1oc2ccccc2c2c1NN1C(=N2)Sc2cc(F)ccc21</smiles>

(7)<smiles></smiles>

(8)

Figure 3. Structure of synthesized compounds (5-8)

Structure from obtained compounds (5-8) was determined from spectral analysis IR, ${ }^{1} \mathrm{H}-\mathrm{NMR}$, mass spectroscopy and elementary analysis. In IR spectra of (5-8) analyzed compounds we can see characteristic bands in region $1710-1727 \mathrm{~cm}^{-1}$ corresponding to $\mathrm{C}=\mathrm{O}$ stretching, $1608-1627 \mathrm{~cm}^{-1}$ there is another band from $\mathrm{C}=\mathrm{N}$ stretching, $1570-1433 \mathrm{~cm}^{-1}$ there is characteristic band for aromatic double bond $\mathrm{C}=\mathrm{C}$ and $\mathrm{SO}_{2}$ vibration are visible in $1270-1122 \mathrm{~cm}^{-1}$.

In ${ }^{1} \mathrm{H}-\mathrm{NMR}$ spectra of gained compounds there are signals in expected positions, signals as multiplets that come from aromatic protons, are in 8.36-7.06 ppm. Among the compounds tested for anti bacterial activity, compound 7 showed highest zone of inhibition against S.aureus in higher concentrations and in lower concentration, the inhibition zone was higher against Aeromonas Salmonicida and Bacillus spp.

\section{Biological activity}

The antimicrobial activity of synthesized compounds (5-8) was determined by cup plate, Kirby-Bauer method. Antimicrobial activity was carried out against $24 \mathrm{~h}$ old cultures of Staphylococcus aureus beta lactamase positive ATCC 25923, collection of WRC (Water Regional Company) from regional hospital, Staphylococcus aureus collection of WRC outside the regional hospital, Streptococcus pneumoniae, Aeromonas salmonicida collection of WRC, Bacillus spp, collection of WRC. The compounds were tested at three concentrations, $0.1 \mathrm{mg} / \mathrm{mL}, 0.3 \mathrm{mg} / \mathrm{mL}$ and $0.5 \mathrm{mg} / \mathrm{mL}$ in dimethylformamide against all organisms. Novobiocin was used as standard drug for antibacterial activity.

Table 1. Microbiological activity of newly synthesized compound

\begin{tabular}{|c|c|c|c|c|c|c|c|c|}
\hline 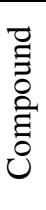 & $\begin{array}{l}\vec{\Xi} \\
\bar{\delta} \\
\tilde{\Xi} \\
\dot{0} \\
\tilde{0} \\
\dot{U}\end{array}$ & 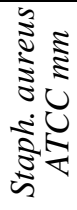 & 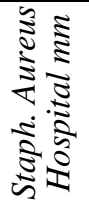 & 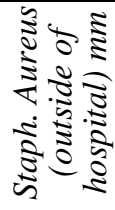 & 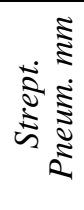 & 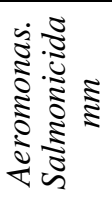 & 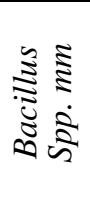 & 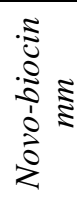 \\
\hline \multirow{4}{*}{5} & 0.1 & 7 & 4 & 3 & 3 & 3 & 3 & 29 \\
\hline & 0.3 & 11 & 3 & 2 & - & 12 & - & 29 \\
\hline & 0.5 & 13 & - & - & 5 & 15 & - & 29 \\
\hline & 0.1 & 2 & 4 & 7 & 9 & 7 & 6 & 29 \\
\hline \multirow{3}{*}{6} & 0.3 & 8 & 4 & 8 & 6 & 13 & - & 29 \\
\hline & 0.5 & 8 & & 10 & 10 & 9 & - & 29 \\
\hline & 0.1 & 7 & 1 & 7 & 1 & 14 & 9 & 10 \\
\hline \multirow[t]{3}{*}{7} & 0.3 & 8 & 3 & 9 & 3 & - & 10 & 10 \\
\hline & 0.5 & 12 & 9 & 14 & 1 & - & - & 10 \\
\hline & 0.1 & 7 & 4 & 3 & 3 & 3 & 3 & 29 \\
\hline \multirow[t]{2}{*}{8} & 0.3 & 11 & 3 & 2 & - & 12 & . & 29 \\
\hline & 0.5 & 13 & - & - & 5 & 15 & - & 29 \\
\hline
\end{tabular}




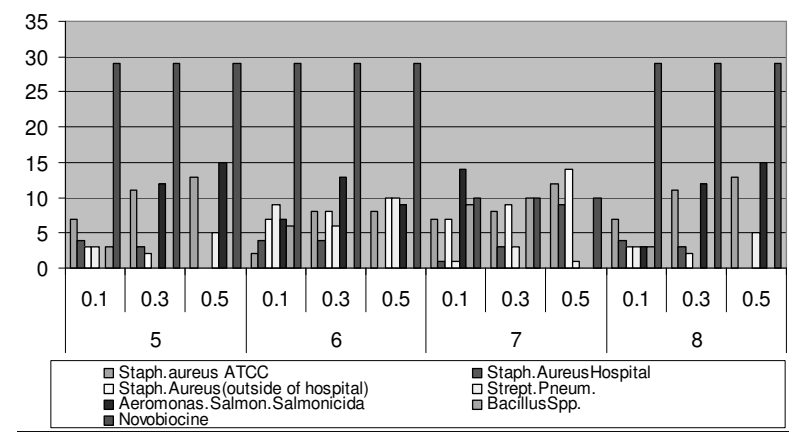

Figure 4. Graphs of microbiological activity results for compounds 5-8

\section{Conclusion}

The present study was aimed at synthesis of some novel chromene-2-one derivatives. The compounds were screened for anti bacterial activities and were found to posse's considerable activity.

\section{References}

1. Govori S, Rapic V, Leci O, Cacic M and Tabakovic I, J Heterocycl Chem., 1996, 33(2), 351-354.

2. Govori S, Kaljaj V, Rapic V, Kaljaj L and Dakovic S, Heterocycl Commun., 2002, 8,129-134.

3. Manolov I and Danchev N D, Eur J Med Chem Chim Ther., 1995, 30(6), 531-536.

4. Arora R B and Mathur C N, Brit J Pharmacol., 1963, 20, 29-35.

5. Ziegler E and Rossmann U, Monatsh Chem., 1957, 88, 25-34.

6. Al-Haiza M A, Mostafa M S and El-Kady M Y, Molecules., 2003, 8(2), 275-286.

7. Dutton C J, Sutcliffe J and Yang B, PCT Int Appl., US1993/006308, 1994.

8 Vogel A I and Tatchell A R, Vogel's Textbook of Practical Organic Chemistry, Hannaford AJ and Smith P W J, $\mathbf{5}^{\text {th }}$ Ed, 1191-1192.

9. Mulwad V V and Shirodkhar J M, Indian J Heterocycl Chem., 2002, 11, 199-202.

10. Spino C, Dodier M and Sotheeswaran S, Bioorg Med Chem Lett., 1998, 8(24), 3475-3478.

11. Thaisrivongs S, Watenpaugh K D, Howe W J, Tomich. P K, Dolak L A, Chong K.T, Tomich C S C, Tomasselli A G, Turner S R, Strohbach J W, Mulichak A M, Janakiraman M N, Moon J B, Lynn J C, Horng M M, Hinshaw R R, Curry K A and Rothrock D J, J Med Chem., 1995, 38, 3624-3637.

12. Gaddam S, Khilevich A, Filer C, Rizzo J D, Giltner J, Flavin M T and Xu Z Q, J Label Comp Radiopharm., 1997, 39, 901-906.

13. Zhao H, Neamati N, Hong H, Mazumder A, Wang S, Sunder S, Milner G W A, Pommier Y and Burke Jr T R, J Med Chem., 1997, 40, 242-249.

14. Gummudavelly S, Sri Ranganathi Y, Bhasker S and Rajkumar N, Asian J Res Chem., 2009, 2, 46-48.

15. Rajeshwar Rao V, Srimanth K and VijayaKumar P, Indian J Heterocycl Chem., 2004, 14, 141-144.

16. Nofal Z M, El-Zahar M I and Abd El-Karim S S, Molecules, 2000, 5, 99-113.

17. Kovac M, Sabatic A and Floch L, Arkivoc, 2001, (vi), 100-108.

18. Tabakovic I, Tabakovic K, Grujic R, Trinajstic N and Meic Z, Heterocycl., 1985, 23(10), 2539-2542

19. Buu-Hoï N P, Martani A, Croisy A, Jacquignon P and Périn F, J Chem Soc C., 1966, 1787-1786. 


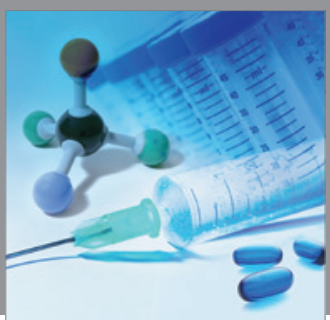

International Journal of

Medicinal Chemistry

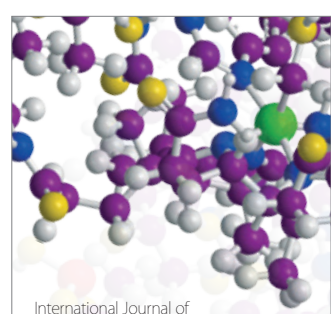

Carbohydrate Chemistry

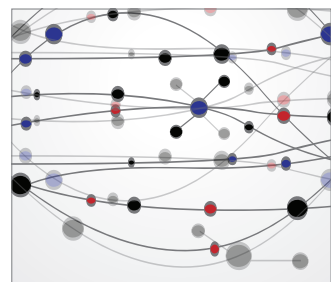

The Scientific World Journal
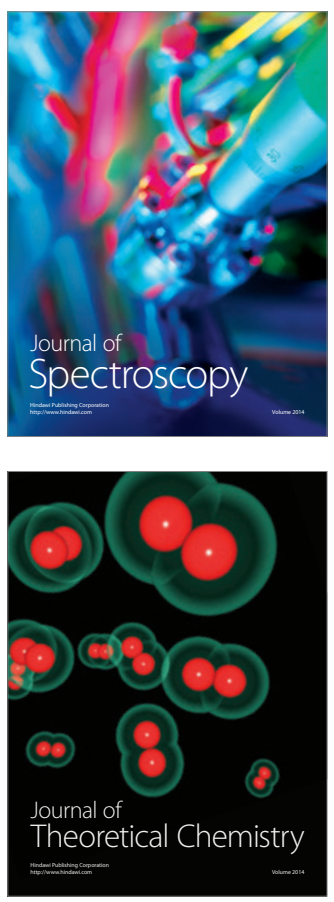
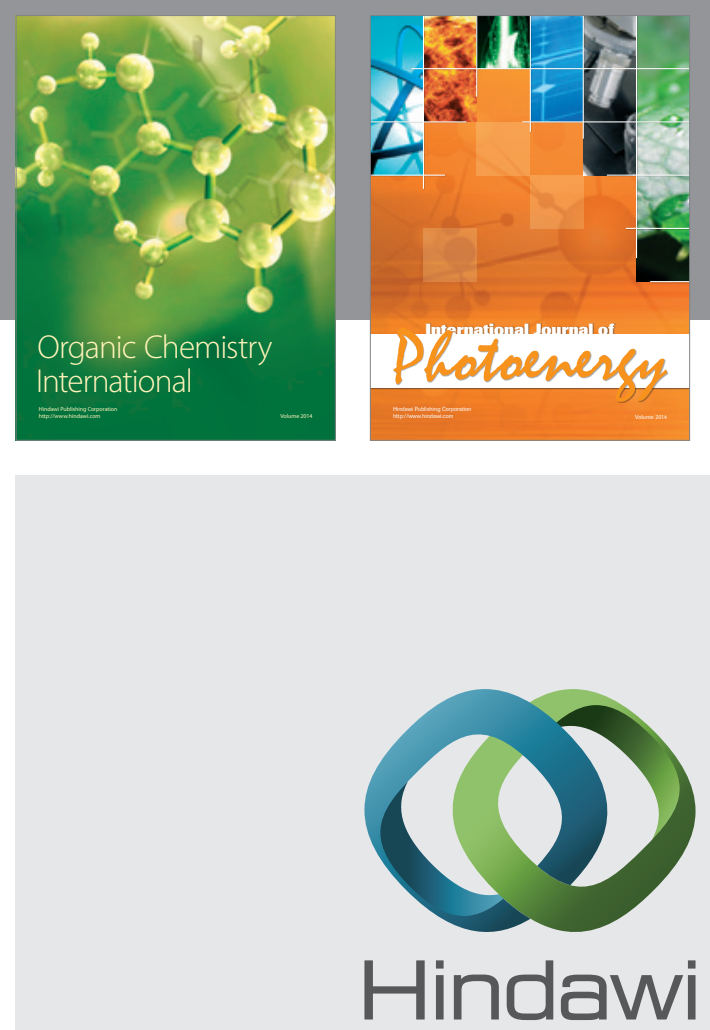

Submit your manuscripts at

http://www.hindawi.com
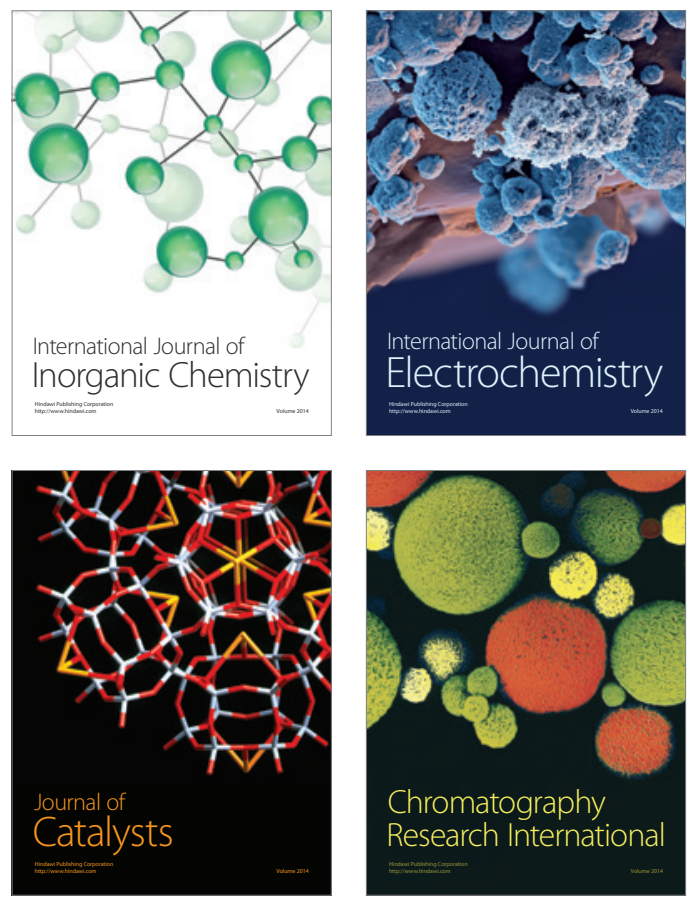
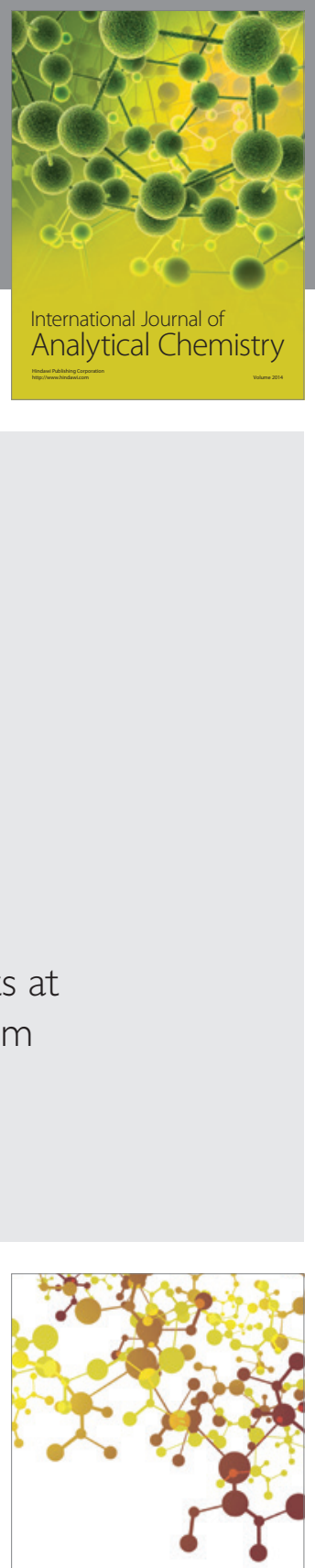

Journal of

Applied Chemistry
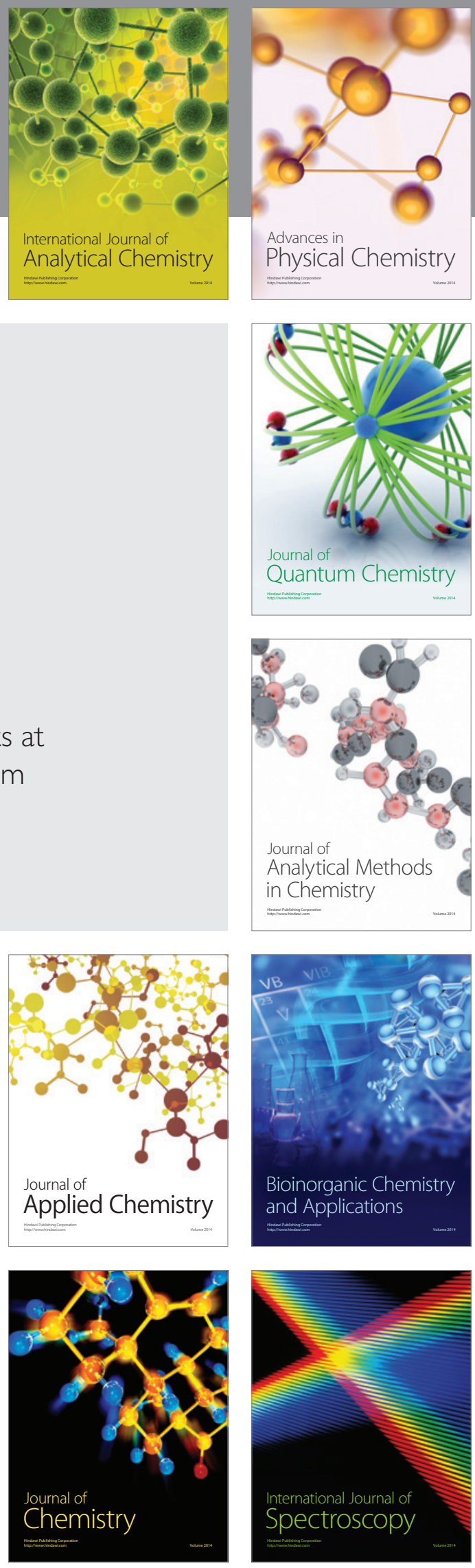